\title{
MESON PHOTOPRODUCTION NEAR THRESHOLD
}

\author{
MADELEINE SOYEUR \\ Département d'Astrophysique, de Physique des Particules, \\ de Physique Nucléaire et de l'Instrumentation Associée \\ Service de Physique Nucléaire \\ Commissariat à l'Energie Atomique/Saclay \\ F-91191 Gif-sur-Yvette Cedex, France
}

\begin{abstract}
These lectures review our understanding of the photoproduction of vector and pseudoscalar mesons from proton targets at incident energies close to the production threshold and at low t. The importance of that particular kinematics to extract information on hadronic vertices is emphasized.
\end{abstract}

\section{Introduction}

The photoproduction of a meson from a proton target is an especially nice process to study. It tells us how a photon passing in the field of a proton transforms into a $q \bar{q}$ pair.

Of course, this process depends strongly on the energy of the photon inducing the reaction, on the momentum transferred to the proton and on the quantum numbers of the meson produced in the final state. In these lectures, we restrict our discussion to photon energies of a few $\mathrm{GeV}$ (typically $\left.\mathrm{E}_{\gamma}<2-3 \mathrm{GeV}\right)$, to small momentum transfers $\left(-\mathrm{t}<1 \mathrm{GeV}^{2}\right)$ and to the low-lying mesons. We consider single meson photoproduction, i. e. the $\gamma p \rightarrow M p$ and the $\gamma p \rightarrow K Y$ reactions, where $p$ denotes a proton, $M$ a meson with no strangeness, $K$ a kaon and $Y$ a hyperon.

In the kinematics defined above, the degrees of freedom involved in the description of meson photoproduction are hadronic. In the long-wavelength limit, the target proton is a source of meson fields. The incident photon can be absorbed by such meson and this interaction produces the final meson (t-channel process). Alternatively, the incident photon can induce the radiative excitation of the target proton into a baryon resonance, which will 
decay into the final meson and a proton or a hyperon (s-channel process). The purpose of meson photoproduction studies near threshold is therefore to learn about hadronic vertices and low-lying baryon resonances.

We will distinguish three types of reactions: the photoproduction of vector mesons $(\omega, \rho, \phi)$, the photoproduction of pseudoscalar mesons $\left(\pi, \eta, \eta^{\prime}\right)$ and the associated photoproduction of a kaon and a hyperon $(\Lambda, \Sigma)$. These three processes are rather different because of the quantum numbers involved.

The threshold energies required to produce the above mesons vary from $0.145 \mathrm{GeV}$ for pions until $1.574 \mathrm{GeV}$ for $\phi$-mesons. The facilities pursuing presently the experimental study of meson photoproduction close to threshold are ELSA at Bonn $\left(\mathrm{E}_{\gamma}=0.5-3.5 \mathrm{GeV}\right)$, MAMI at Mainz $\left(\mathrm{E}_{\gamma}=0.05-0.8\right.$ $\mathrm{GeV}$ ) and Jefferson Laboratory at Newport News $\left(\mathrm{E}_{\gamma}=0.2-5.2 \mathrm{GeV}\right)$. A limited program is also pursued with GRAAL at Grenoble $\left(\mathrm{E}_{\gamma}=0.35-1.5\right.$ $\mathrm{GeV})$.

This manuscript is organized as follows. Section 2 recalls briefly the variables and the basic processes underlying the theoretical description of meson photoproduction, together with their Feynman graph representation. Section 3 is devoted to the photoproduction of vector mesons and Section 4 to the photoproduction of pseudoscalar mesons with $\mathrm{S}=0$. The associated photoproduction of a kaon and a hyperon is presented in Section 5. We conclude by a few remarks in Section 6 .

\section{Kinematics and s- and t-channel amplitudes}

A general amplitude for the $\gamma\left(q_{1}\right)+p\left(p_{1}\right) \rightarrow M\left(q_{2}\right)+B\left(p_{2}\right)$ reaction (M is a meson and $\mathrm{B}$ a proton or a hyperon) is pictured in Fig. 1.

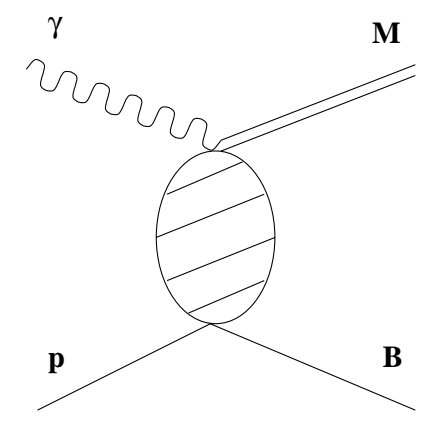

Figure 1. Meson photoproduction from a proton target.

The Lorentz-invariant Mandelstam variables characterizing the kinematics of the process illustrated in Fig. 1 are defined by 


$$
\begin{aligned}
& s=\left(q_{1}+p_{1}\right)^{2}=\left(q_{2}+p_{2}\right)^{2}, \\
& t=\left(q_{1}-q_{2}\right)^{2}=\left(p_{2}-p_{1}\right)^{2}, \\
& u=\left(p_{1}-q_{2}\right)^{2}=\left(p_{2}-q_{1}\right)^{2} .
\end{aligned}
$$

The value of $\sqrt{s}$ gives the total energy in the center of mass system. The variable $t$ represents the momentum transfer. This quantity is negative in the processes we consider. It is useful to define $Q^{2} \equiv-t$. The variable $u$ is appropriate to discuss the crossed graphs introduced below.

The theoretical descriptions of the $\gamma+p \rightarrow M+B$ reactions emphasized in these lectures are based on the resonance approximation [1] to the scattering matrix associated with the amplitude of Fig. 1.

In the t-channel, the amplitude is dominated by meson-exchanges (Fig. 2 ). In the s-channel, it is approximated by the exchange of baryon resonances (in addition to the nucleon pole). The s-channel diagrams involve both direct and crossed terms (Figs. 3 and 4 respectively).

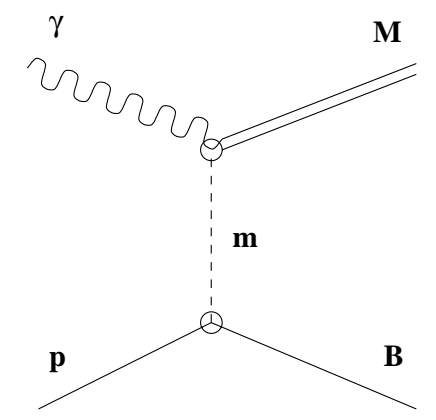

Figure 2. Diagram representing a meson-exchange term in the t-channel.

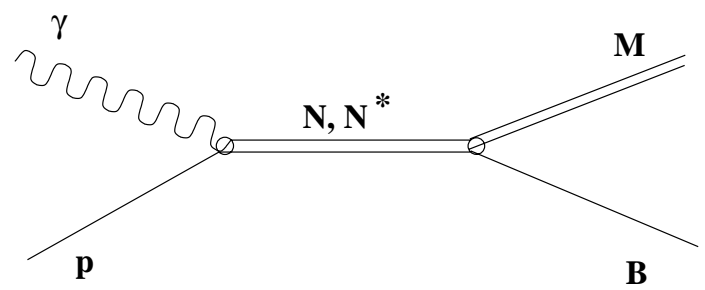

Figure 3. Diagram representing direct terms in the s-channel with intermediate nucleon and baryon resonances.

Duality implies that the scattering amplitude can be written as a complete sum of resonances either in the s- or in the t-channel [2]. In practice, 


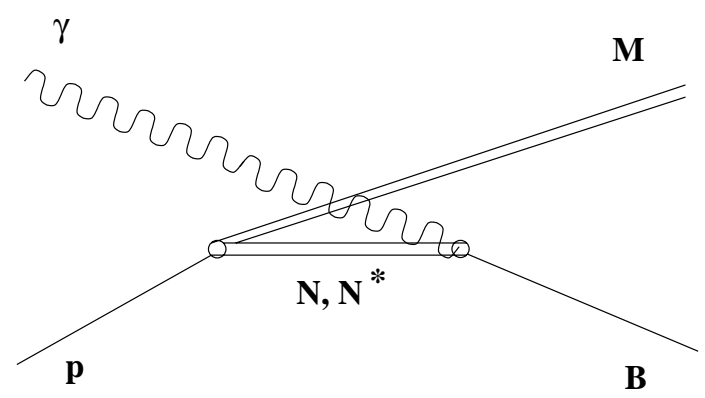

Figure 4. Diagram representing crossed-terms in the s-channel with intermediate nucleon and baryon resonances.

one assumes that only a few resonances contribute to a specific process. Both s- and t-channel diagrams are then added. There is no justification for this procedure, in principle likely to induce double-counting. The practical rule is to consider the exchanges of resonances strongly coupled to the initial and final channels. In the case of t-channel diagrams, the exchange of the lightest mesons always dominates at low $\mathrm{Q}^{2}$ because of the form of the meson propagator $\left[-\mathrm{Q}^{2}-\mathrm{m}_{m}^{2}\right]^{-1}$.

In order to obtain instructive constraints on hadronic vertices from studies of meson photoproduction from protons, it proves important to choose kinematics in which these processes are dominated by a very small number of diagrams. We will show in the next sections that incident energies close to the production threshold and low momentum transfers are required both for the validity of the description outlined above and for extracting relevant information from the data.

It is worth noticing that very complementary studies of hadronic vertices and baryon resonances can be made using similar and consistent descriptions of pion induced meson production off proton targets near threshold, i. e. the $\pi^{-} p \rightarrow M N$ and the $\pi^{-} p \rightarrow K Y$ reactions.

\section{The photoproduction of vector mesons}

We restrict our discussion to the photoproduction of the three low-lying neutral vector mesons, the $\rho^{0}(770)$, the $\omega(782)$ and the $\phi(1019)$. They are of special interest in photoproduction processes because they have the same quantum numbers as the photon $(\mathrm{J}=1, \mathrm{I}=0$ or $1, \mathrm{P}=-1, \mathrm{C}=-1)$. They are also related dynamically to the photon through the current-field identities of Kroll, Lee and Zumino [3]. These identities relate the electromagnetic 
current to the neutral vector meson fields,

$$
\mathcal{J}_{\mu}^{e m}(I=1)=-\frac{e m_{\rho}^{2}}{2 g_{\rho}} \rho_{\mu},
$$

and

$$
\mathcal{J}_{\mu}^{e m}(I=0)=-\frac{e m_{\omega}^{2}}{2 g_{\omega}} \omega_{\mu}-\frac{e m_{\phi}^{2}}{2 g_{\phi}} \phi_{\mu}
$$

for the isovector and isoscalar parts of the current respectively. In the above equations, $m_{\rho}, m_{\omega}$ and $m_{\phi}$ are the $\rho$-, $\omega$ - and $\phi$-masses and $g_{\rho}, g_{\omega}$ and $g_{\phi}$ are dimensionless constants, which can be determined from the $e^{+} e^{-}$partial decay widths of the $\rho^{-}, \omega^{-}$and $\phi$-mesons [4] to be

$$
\begin{gathered}
g_{\rho}^{2}=6.3, \\
g_{\omega}^{2}=72.7,
\end{gathered}
$$

and

$$
g_{\phi}^{2}=41
$$

Eqs. (4) and (5) are the simplest form of the Vector Dominance assumption [5] and provide a model to describe the coupling of virtual photons to hadrons at values of $\left|\mathrm{q}^{2}\right|<1 \mathrm{GeV}^{2}$. They imply that virtual photons couple to hadrons through intermediate vector mesons of appropriate quantum numbers, the conversion of photons into vector mesons being proportional to the quantities $f_{V}=e m_{V}^{2} / 2 g_{V}$.

The successes and failures of this simple Vector Dominance model in reproducing hadron form factors are summarized in Ref. [6] (see also Ref. [7]). This model is very successful in describing the pion form factor in both time-like and space-like regions. The electromagnetic form factor of the pion is entirely given by an intermediate $\rho$-meson coupled to a pointlike pion (except for a small G-parity violating contribution from the $\omega$ meson). The Vector Dominance model is also capable of describing the data available on the electromagnetic structure of the $\eta$ and $\eta^{\prime}$-mesons. The electromagnetic structure of vector mesons and of baryons appears more complicated. Their electromagnetic size can but be described by twocomponent models combining Vector Dominance and an intrinsic hadronic form factor (see Ref. [8] for a discussion of the $\omega(782) \rightarrow \pi^{0} \mu^{+} \mu^{-}$transition form factor and Refs. [9, 10] for models of the nucleon form factors). Of particular importance for the discussion of the photoproduction of vector mesons is the $\omega \rightarrow \pi^{0} \mu^{+} \mu^{-}$form factor because it characterizes the $\omega \pi \rho$ vertex. Attempts to understand the available data on this process [11] have met with little success. The relation of the $\omega \pi \rho$ vertex to chiral anomalies makes it particularly important to have more accurate data on this decay. 
The theoretical description of vector meson photoproduction from proton targets near threshold is expected to evolve very much in the coming years. The data presently available $[12,13,14]$ date from the late sixties and the early seventies. New data have been taken with the SAPHIR detector at ELSA (Bonn) [15] and an extensive program has been carried very recently at Jefferson Laboratory with the CLAS detector [16]. The results of these experiments are not yet finalized. They should lead to a much more detailed understanding of the dynamics of vector meson photoproduction than the limited data now available to test models.

The present feeling is that the photoproduction of vector mesons close to threshold is a process dominated by t-channel exchanges $[8,17]$. Because photons and vector mesons behave similarly under charge conjugation transformations $(\mathrm{C}=-1)$, the only mesons which can be exchanged in diagrams of the kind shown in Fig. 2 must have $\mathrm{C}=+1$. They are either pseudoscalar $\left(\pi^{0}, \eta\right)$ or scalar $(\sigma$-like).

\subsection{THE PHOTOPRODUCTION OF $\omega$-MESONS}

The t-channel meson-exchange description of the $\gamma p \rightarrow \omega p$ reaction near threshold $\left[\mathrm{E}_{\gamma}^{\text {thresh }}=1.108 \mathrm{GeV}\right]$ appears particularly suited because the Particle Data Group [4] does not list any established baryon resonance decaying into the $\omega N$ channel which would generate a significant s-channel contribution. The possible pseudoscalar and scalar t-channel exchange contributions to the $\gamma p \rightarrow \omega p$ reaction near threshold are displayed in Fig.

5 .

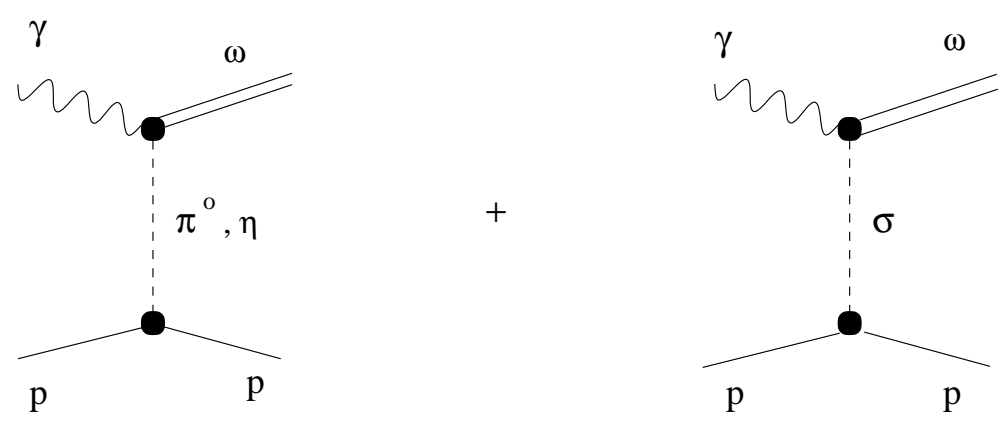

Figure 5. t-channel exchange contributions to the $\gamma p \rightarrow \omega p$ reaction near threshold.

The relative importance of these contributions can be assessed from the partial radiative decay widths of the $\omega$-meson [4],

$$
, \omega \rightarrow \pi^{0} \gamma=(715 \pm 42) \mathrm{keV},
$$




$$
\begin{gathered}
, \omega \rightarrow\left(\pi^{0} \pi^{0}\right)_{\sigma \gamma}=(0.6 \pm 0.2) \mathrm{keV}, \\
, \omega \rightarrow \eta \gamma=(5.5 \pm 0.8) \mathrm{keV},
\end{gathered}
$$

and from the meson-nucleon coupling constants $[18,19]$

$$
\begin{aligned}
g_{\pi N N}^{2} / 4 \pi & \simeq 14, \\
g_{\sigma N N}^{2} / 4 \pi & \simeq 8, \\
g_{\eta N N}^{2} / 4 \pi & \simeq 0.4 .
\end{aligned}
$$

It is clear from these couplings that the $\gamma p \rightarrow \omega p$ reaction near threshold will be dominated by $\pi$-exchange. The calculation of Ref. [8] shows actually that the available data [12] on the $\omega$ photoproduction from protons for $\mathrm{E}_{\gamma}<2 \mathrm{GeV}$ can be well understood assuming that the reaction is entirely driven by pion-exchange. The corresponding graph in the Vector Dominance Model is given in Fig. 6 . This conclusion rests on the choices made for the $\omega \pi \rho$ and $\pi N N$ form factors. The $\omega \pi \rho$ form factor is chosen so as to reproduce the $\omega \rightarrow \pi^{0} \mu^{+} \mu^{-}$transition form factor discussed previously. The $\pi N N$ form factor is parametrized by a monopole form with a cutoff $\Lambda_{\pi}=700 \mathrm{MeV}$, as suggested by recent analyses [20]. The model should be valid for $-\mathrm{t} \leq \Lambda_{\pi}^{2} \simeq 0.5 \mathrm{GeV}^{2}$.

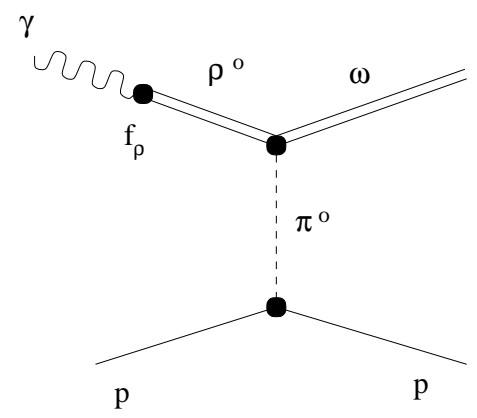

Figure 6. t-channel $\pi$-exchange amplitude for the $\gamma p \rightarrow \omega p$ reaction near threshold in the Vector Dominance Model.

The one-pion-exchange description of the $\gamma p \rightarrow \omega p$ reaction near threshold $[8]$ is shown in Figs. 7 and 8.

From Fig. 7 , it can be seen that the $\pi$-exchange model accounts for the data below $2 \mathrm{GeV}$, while for larger photon energies, an additional contribution is needed. The missing cross section is almost independent of energy, a behaviour consistent with the Pomeron-exchange term becoming dominant. Fig. 8 indicates that the general trend of the $Q^{2}$-dependence is well 


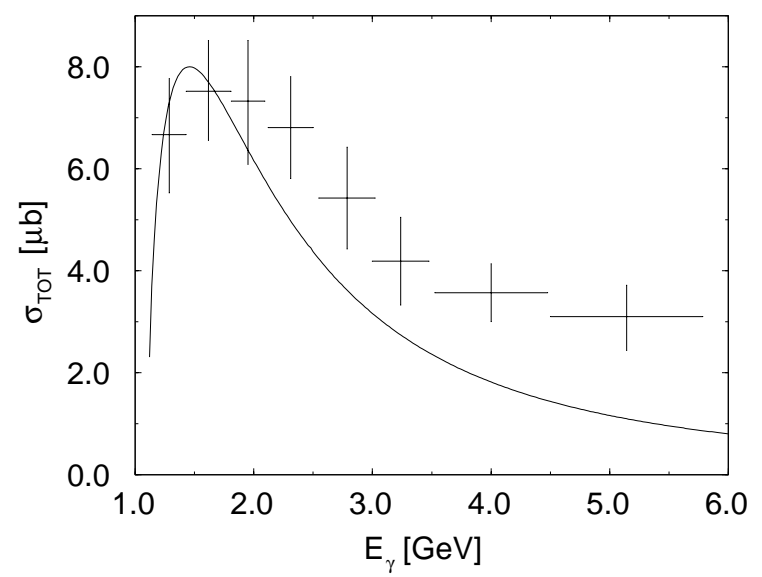

Figure 7. Energy dependence of the one-pion-exchange description of the $\gamma p \rightarrow \omega p$ cross section (from Ref. [8]). The data are those of Ref. [12].

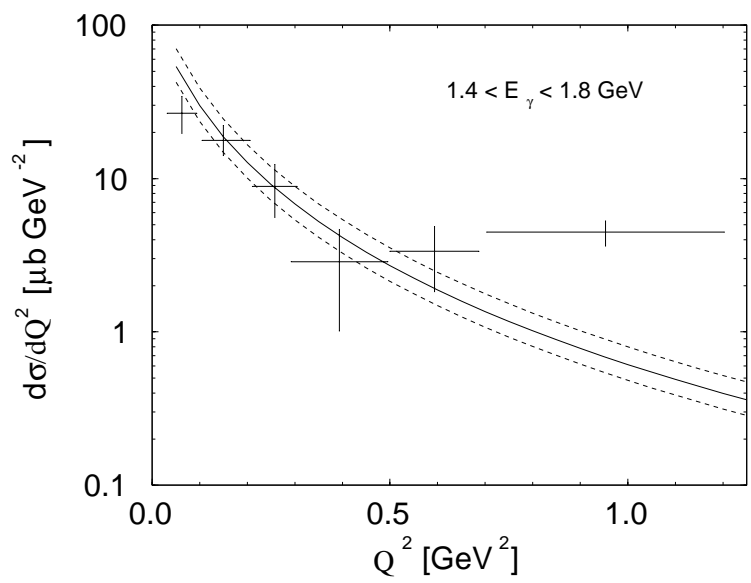

Figure 8. Differential cross section $d \sigma / d Q^{2}$ for the $\gamma p \rightarrow \omega p$ reaction at $1.4<E_{\gamma}<$ $1.6 \mathrm{GeV}$. The data are from Ref. [12]. The full line is the $\pi$-exchange model at $E_{\gamma}=1.6$ $\mathrm{GeV}$ (from Ref. [8]). The dotted lines show the uncertainties due to the photon energy resolution.

reproduced for $Q^{2}<0.5 G e V^{2}$, i. e. within the expected range of validity of the model.

For the range of photon energies investigated $\left(E_{\gamma}<2 \mathrm{GeV}\right)$, there is no clear indication from the data of Ref. [12] of anything else contributing to the $\gamma p \rightarrow \omega p$ process in the t-channel but the $\pi$-exchange. It is quite remarkable that for values of the coupling constants and of the form factors consistent with other processes, the total cross section is correctly given by 
this simple model.

New data from SAPHIR [15] and CLAS [16] may however require other contributions than the single $\pi$-exchange discussed above. Preliminary results suggest that the differential cross section $d \sigma / d Q^{2}$ for the $\gamma p \rightarrow \omega p$ reaction very close to threshold $\left(E_{\gamma}<1.3 \mathrm{GeV}\right)$ does not show the characteristic $Q^{2}$-dependence of a meson-exchange amplitude. If these data are confirmed, they would unravel new and very interesting physics.

\subsection{THE PHOTOPRODUCTION OF $\rho^{0}$-MESONS}

Eventhough the t-channel meson-exchange discussed above for the $\gamma p \rightarrow \omega p$ reaction near threshold $\left[\mathrm{E}_{\gamma}^{\text {thresh }}=1.086 \mathrm{GeV}\right]$ appears less justified for $\rho^{0}-$ meson photoproduction (there are low-lying baryon resonances decaying into the $\rho$-nucleon channel [4]), it is interesting to see how well such model can reproduce the available data [12].

The possible pseudoscalar and scalar t-channel exchange contributions to the $\gamma p \rightarrow \rho^{0} p$ reaction near threshold are displayed in Fig. 9
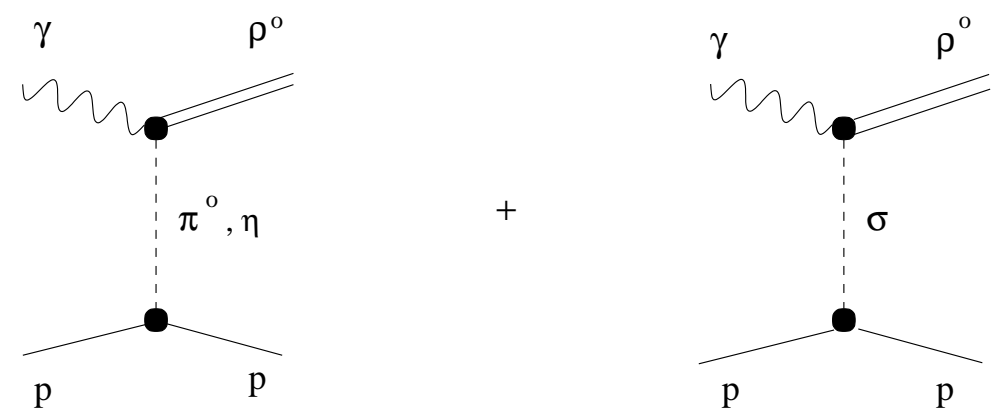

Figure 9. t-channel exchange contributions to the $\gamma p \rightarrow \rho^{0} p$ reaction near threshold.

In contrast to what happens in the $\omega$ photoproduction, the scalarexchange appears to be the dominant contribution to the $\gamma p \rightarrow \rho^{0} p$ reaction while the pion-exchange plays a minor role [8]. This can again be inferred from the partial radiative decay widths of the $\rho^{0}$-meson [4],

$$
\begin{gathered}
, \rho^{0} \rightarrow \pi^{0} \gamma=(102 \pm 26) \mathrm{keV}, \\
, \rho^{0} \rightarrow\left(\pi^{+} \pi^{-}\right)_{\sigma \gamma}=(1492 \pm 241) \mathrm{keV}, \\
, \rho^{0} \rightarrow \eta \gamma=(36 \pm 13) \mathrm{keV},
\end{gathered}
$$

and from the meson-nucleon coupling constants given in Eqs. (12)-(14). 
Both $\pi$ - and $\sigma$-exchanges have been included in the calculation of the $\gamma p \rightarrow \rho^{0} p$ cross section [8] (the $\eta$-exchange is negligible). These terms are shown in the Vector Dominance Model in Fig. 10.

$\gamma$

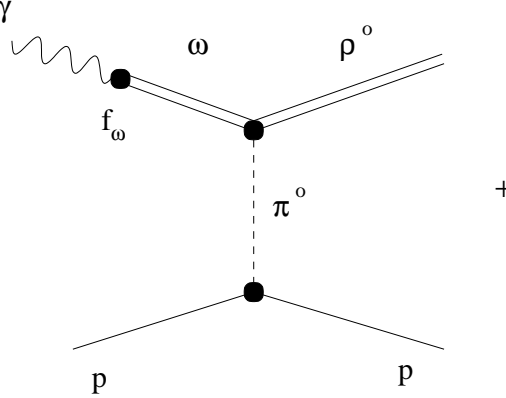

$\gamma$

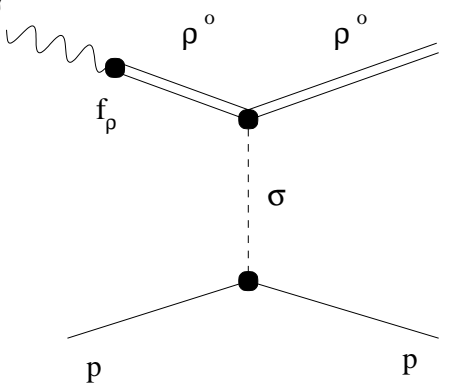

Figure 10. t-channel $(\pi+\sigma)$-exchange contributions to the $\gamma p \rightarrow \rho^{0} p$ reaction near threshold in the Vector Dominance Model.

The $\pi$-exchange contribution is evaluated with the same parameters as those used for the description of the $\gamma p \rightarrow \omega p$ cross section. The effective $\sigma$-exchange $\left(m_{\sigma}=500 \mathrm{MeV}\right)$ represents the exchange of two uncorrelated as well as two resonating pions. The details of the $\sigma \mathrm{NN}$ and of the $\rho^{0} \sigma \rho^{0}$ vertices are given in Ref. [8]. The interesting result is that, for expected values of the form factor cutoffs (of the order of $1 \mathrm{GeV}$ ), both the total and differential cross sections [12] can be reproduced by a $(\pi+\sigma)$-exchange model in the expected range of validity of such model $\left(E_{\gamma}<2 \mathrm{GeV},-\mathrm{t} \leq 0.5\right.$ $\mathrm{GeV}^{2}$ ). This is illustrated in Figs. 11 and 12. Again, and within large error bars, the missing cross section at higher photon energies appears almost independent of energy, suggesting a Pomeron-exchange contribution beyond $E_{\gamma} \simeq 2 \mathrm{GeV}$.

Is the effective $\sigma$-exchange the right dynamical description of the $\rho^{0}$ meson photoproduction from protons near threshold? Forthcoming data of the CLAS Collaboration [16] should be very helpful in clarifying this issue.

\subsection{THE PHOTOPRODUCTION OF $\phi$-MESONS}

The photoproduction of $\phi$-mesons near threshold $\left[\mathrm{E}_{\gamma}^{\text {thresh }}=1.574 \mathrm{GeV}\right]$ appears as a more complicated process than the photoproduction of $\omega$ - and $\rho^{0}$-mesons discussed in the previous subsections. The $\gamma p \rightarrow \phi p$ reaction cross section seem to result, even at threshold, from a large number of processes [17].

As for the $\omega$ - and $\rho^{0}$-mesons, we expect t-channel meson exchanges. The relevant $\phi$ radiative decay widths are [4] 


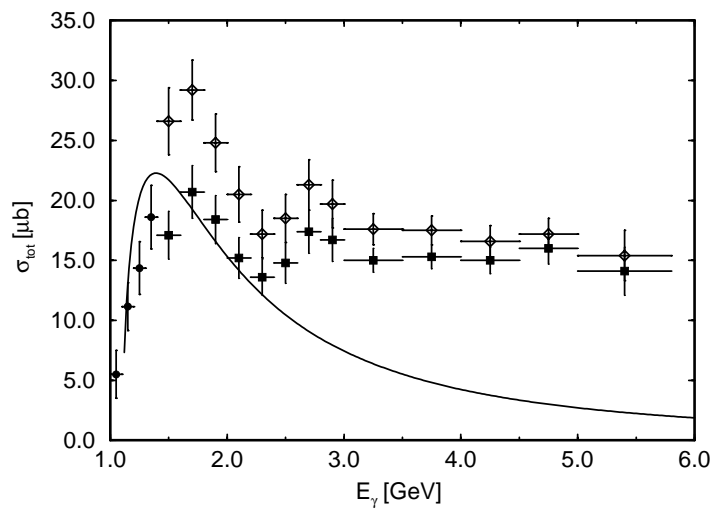

Figure 11. Energy dependence of the $(\pi+\sigma)$-exchange model of the $\gamma p \rightarrow \rho^{0} p$ total cross section (from Ref. [8]). The data are those of Ref. [12].

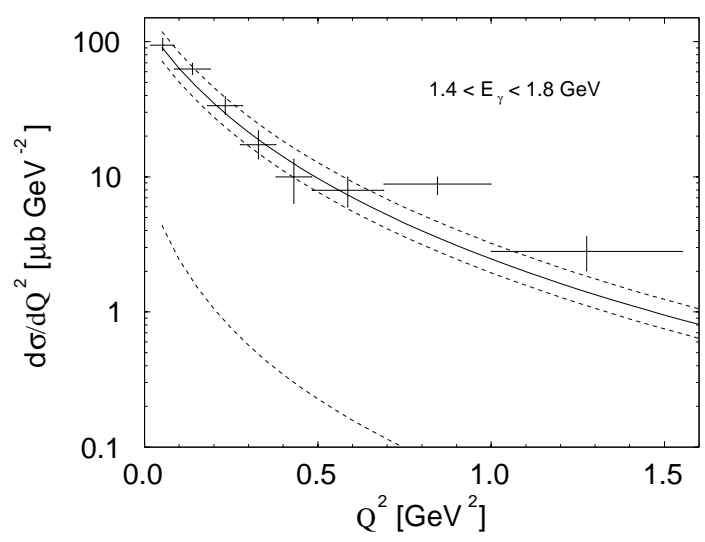

Figure 12. Differential cross section $d \sigma / d Q^{2}$ for the $\gamma p \rightarrow \rho^{0} p$ reaction (from Ref. [8]). The data are from Ref. [12]. The full line is the $(\pi+\sigma)$-exchange model for $E_{\gamma}=1.6$ $\mathrm{GeV}$. The dashed line shows the $\pi$-exchange contribution. The dotted lines indicate the uncertainties due to the photon energy resolution.

$$
\begin{aligned}
& { }_{\phi \rightarrow \pi^{0} \gamma}=(5.8 \pm 0.6) \mathrm{keV},
\end{aligned}
$$

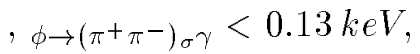

$$
\begin{aligned}
& { }_{\phi \rightarrow \eta \gamma}=(55.8 \pm 2.6) \mathrm{keV} \text {. }
\end{aligned}
$$


Together with the meson-nucleon coupling constants given in Eqs. (12)(14), these radiative decay widths suggest that only the $\pi^{0}$ - and $\eta$-exchanges will play a role. They are shown in the Vector Dominance Model in Fig. 13.

$\gamma$

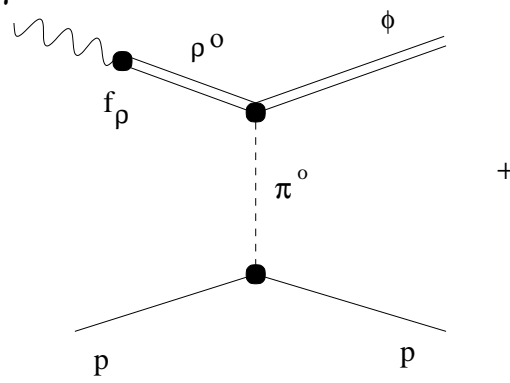

$\gamma$

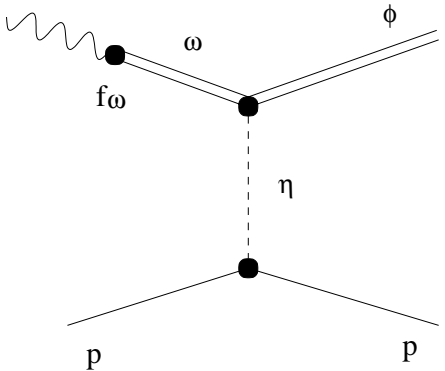

Figure 13. t-channel exchange contributions to the $\gamma p \rightarrow \phi p$ reaction near threshold in the Vector Dominance Model.

The calculation of these diagrams is done in complete analogy to the computation of the $\pi$-exchange amplitude for the $\gamma p \rightarrow \omega p$ reaction (illustrated in Fig. 6). We note however that the $\pi$ - and $\eta$-exchange amplitudes interfer in this case. The form of the effective Lagrangians and the values of the coupling constants and cutoff parameters are discussed in Ref. [17]. The main result of this calculation is that the $(\pi+\eta)$-exchange model does not reproduce at all the data [14] at $E_{\gamma}=2 \mathrm{GeV}$ [17]. The corresponding cross section is only 20 percent of the measured cross section at low $t$.

In addition to t-channel meson exchanges, the model developed in Ref. [17] contains two Pomeron trajectories, one with a positive intercept $\left(\mathrm{P}_{1}\right)$ and one with a negative intercept $\left(\mathrm{P}_{2}\right)$. They provide most of the $\gamma p \rightarrow \phi p$ reaction cross section, as illustrated in Fig. 14.

Data taken recently on the $\gamma p \rightarrow \phi p$ reaction at Jefferson Laboratory in a broad incident energy range with the CLAS detector [16] should help in undertanding the nature of Pomeron trajectories which seem to dominate the photoproduction of $\phi$-mesons from proton targets, even very close to threshold.

\section{The photoproduction of pseudoscalar mesons $(\mathrm{S}=0)$}

We review briefly in this Section the photoproduction of pions, $\eta_{\text {- }}$ and $\eta^{\prime}$-mesons. The quantum numbers of the $\pi^{0}-, \eta^{-}$and $\eta^{\prime}$-mesons differ from those of the photon by which they are produced. These mesons have no spin $(\mathrm{J}=0)$ and their transformation under charge conjugation invariance is characterized by $\mathrm{C}=+1$. This implies that the t-channel exchanges contributing 


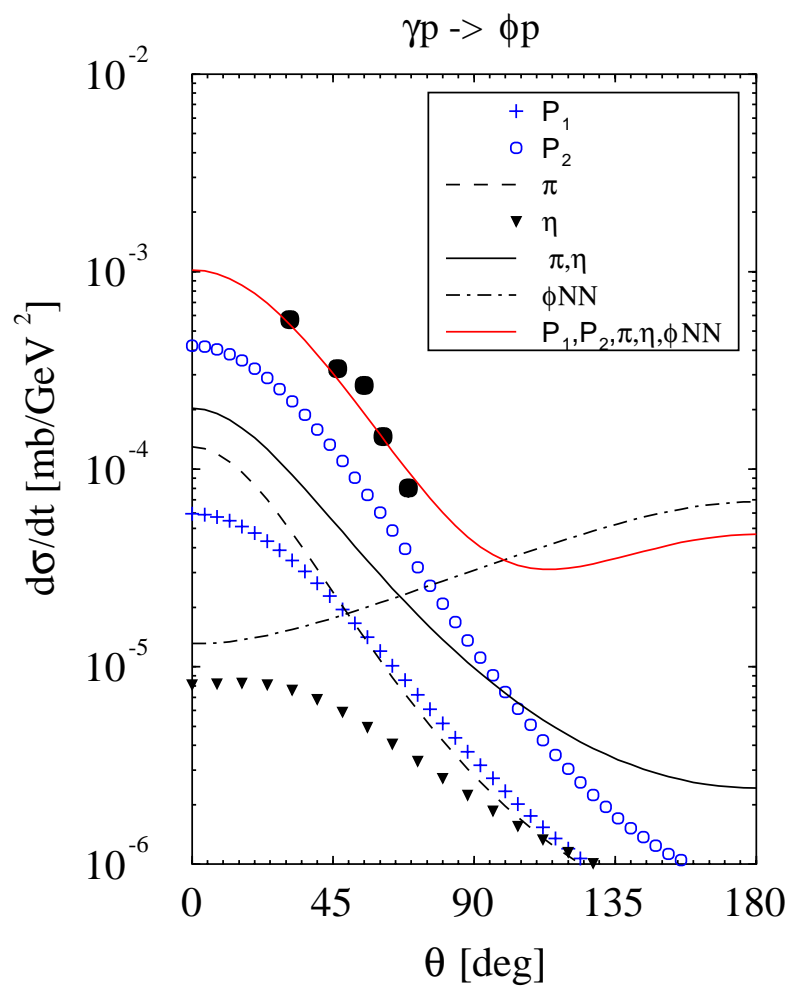

Figure 14. Differential cross section for the $\gamma p \rightarrow \phi p$ reaction at $E_{\gamma}=2 \mathrm{GeV}$. The data are from Ref. [14] and the theoretical curves from Ref. [17]. The lower full curve is the $(\pi+\eta)$-exchange contribution. The $\mathrm{P}_{1}$ and $\mathrm{P}_{2}$ Pomeron terms are indicated by crosses and circles respectively. The upper full curve is the total theoretical differential cross section.

to the $\gamma p \rightarrow \pi^{0} p, \gamma p \rightarrow \eta p$ and $\gamma p \rightarrow \eta^{\prime} p$ reactions will be restricted to the exchange of vector mesons $\left(\rho^{0}, \omega\right)$, which have $\mathrm{C}=-1$.

\subsection{THE PHOTOPRODUCTION OF PIONS}

In what follows, we will distinguish the photoproduction of pions very close to threshold $\left[\mathrm{E}_{\gamma}^{\text {thresh }}=0.145 \mathrm{GeV}\right]$, i.e. $150<E_{\gamma}<400 \mathrm{MeV}$, and their photoproduction further from threshold, i.e. $400 \mathrm{MeV}<E_{\gamma}<1 \mathrm{GeV}$. In the lower energy range, the dynamics of pion photoproduction is governed by t-channel meson exchanges, the Kroll-Ruderman term and s-channel contributions with intermediate nucleon and $\Delta(1232)$ resonance. Above 400 $\mathrm{MeV}$, many more s-channel contributions involving baryon resonances with large $\pi \mathrm{N}$ decay widths are needed to understand the data. 


\subsubsection{The photoproduction of $\pi$-mesons very close to threshold}

We discuss first the photoproduction of positively charged pions. The lowest order terms are shown in Figs. 15-18.

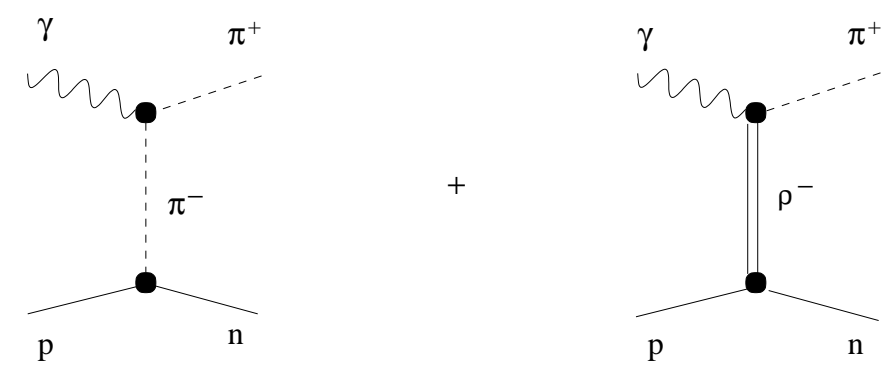

Figure 15. t-channel pion pole term and $\rho^{-}$-exchange contributions to the $\gamma p \rightarrow \pi^{+} n$ reaction near threshold.

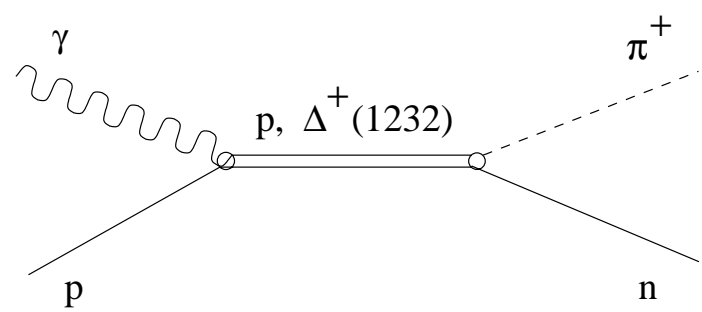

Figure 16. Direct s-channel exchange contributions to the $\gamma p \rightarrow \pi^{+} n$ reaction near threshold.

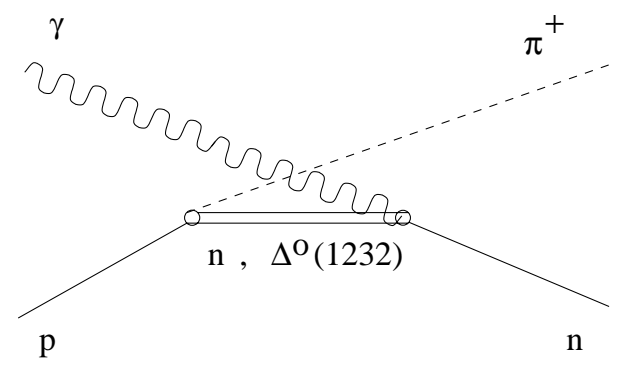

Figure 17. Crossed s-channel exchange contributions to the $\gamma p \rightarrow \pi^{+} n$ reaction near threshold. 


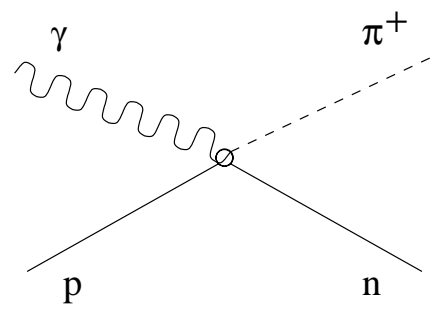

Figure 18. Kroll-Ruderman contribution to the $\gamma p \rightarrow \pi^{+} n$ reaction near threshold.

The study of the $\gamma p \rightarrow \pi^{+} n$ reaction near threshold is now textbook physics. We will not go through the computation of the different diagrams in detail but simply comment on their respective importance. We refer to the book of Ericson and Weise for a thorough discussion of each term [21].

The pion pole term (the left-hand graph of Fig. 15) is allowed for charged pion production only. It vanishes at threshold but becomes important for larger $\mathrm{E}_{\gamma}$. The $\rho$-exchange term (the right-hand graph of Fig. 15) is rather small as a consequence of the low partial decay width of the $\rho$-meson into the $\pi \gamma$ channel discussed previously.

The s-channel nucleon and $\Delta(1232)$ contributions (Figs. 16 and 17 ) are responsible for the production of $\pi^{+} n$ final states in relative p-wave. They are driven by magnetic interactions.

The Kroll-Ruderman term (Fig. 18) is a consequence of gauge invariance. Its form is given by the minimal coupling prescription. It contributes to charged pion photoproduction only. It is dominant at threshold and leads to the production of $\pi^{+} n$ final states in relative s-wave.

The photoproduction of neutral pions, the $\gamma p \rightarrow \pi^{0} p$ process is induced by t-channel terms restricted to vector-meson exchanges and s-channel terms analogous to those involved in charged pion photoproduction (with appropriate charges).

The role of the different terms contributing to pion photoproduction near threshold can be best understood by performing multipole expansions of the photoproduction amplitudes. The electric and magnetic multipoles are denoted by $\mathrm{E}_{l \pm}$ and $\mathrm{M}_{l \pm}$, where $l$ is the orbital angular momentum of the pion and the \pm sign is related to the total pion-nucleon spin $\mathrm{j}=|l \pm 1 / 2| \equiv l \pm$ [21].

In the charged pion photoproduction process $\left(\gamma p \rightarrow \pi^{+} n\right)$, the electric and magnetic dipoles $\mathrm{E}_{0+}$ and $\mathrm{M}_{1+}$ dominate. The $\mathrm{E}_{0+}$ multipole arises from the t-channel pion exchange and the Kroll-Ruderman term. The $\mathrm{M}_{1+}$ multipole comes from the s-channel graphs. In the neutral pion photoproduction process $\left(\gamma p \rightarrow \pi^{0} p\right)$, the $\mathrm{M}_{1+}$ multipole is the only contribution. We 
refer to Ref. [21] for a detailed discussion. We illustrate the main result in Fig. 19 by comparing a recent calculation (involving constraints from fixed t dispersion relations and unitarity) [22] to the existing data $[23,24,25,26]$. For completeness, we show all isospin channels.
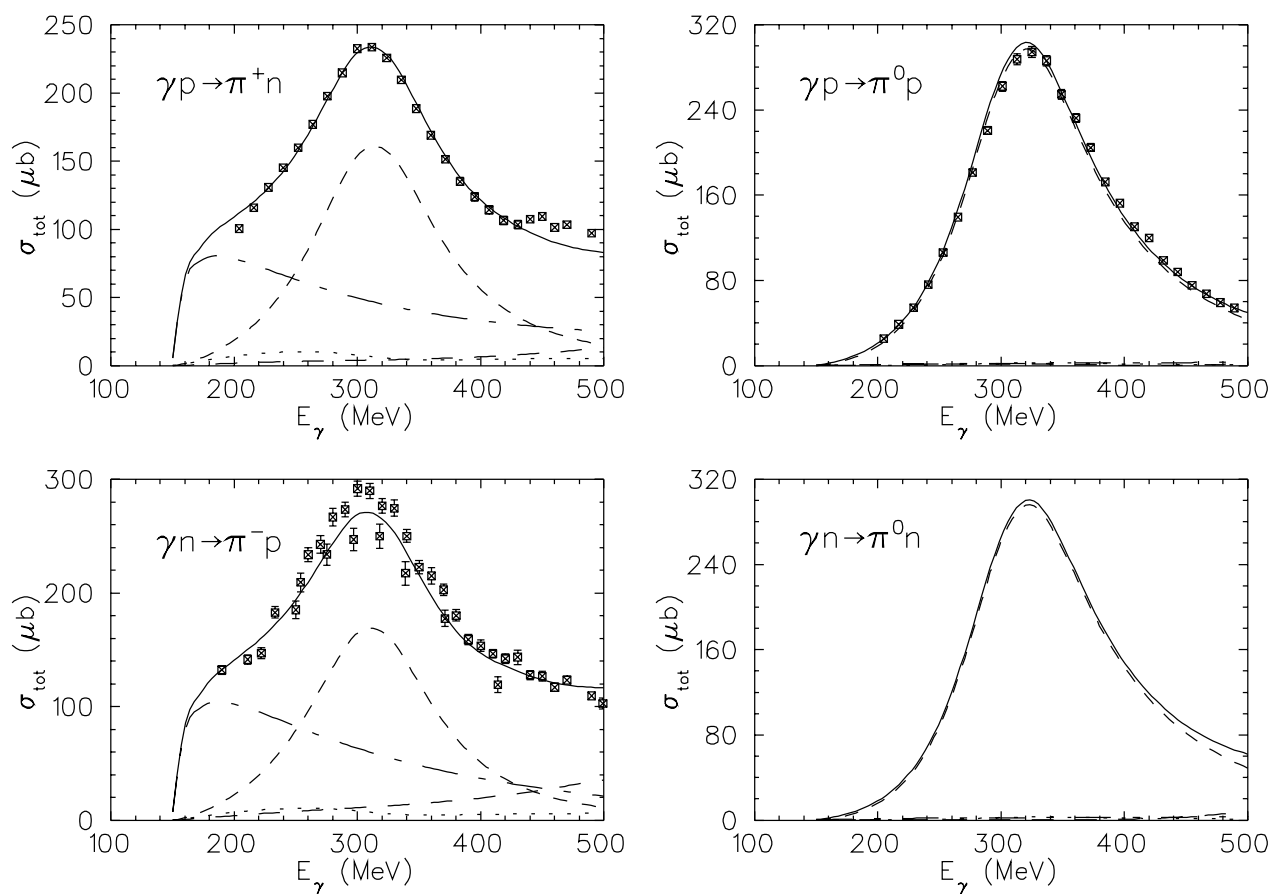

Figure 19. Calculated total cross sections for pion photoproduction from proton and neutron targets [22]. The contributions of the leading multipoles are shown: $\mathrm{M}_{1+}$ (dashed lines), $\mathrm{E}_{0+}$ (dashed-dotted lines), $\mathrm{M}_{1-}$ (long dashed lines), $\mathrm{E}_{1+}$ (dotted lines). The data are from Refs. [23-26].

There are many interesting issues in the study of pion photoproduction from nucleon target very close to threshold which are not reviewed here. One of them is the determination of the ratio of the electric quadrupole and magnetic dipole excitations of the $\Delta(1232)$ [27]. Another recent development is to use the photoproduction of neutral pions from the proton at low energy to test Chiral Perturbation Theory [28].

\subsubsection{The photoproduction of $\pi$-mesons further from threshold}

For $400 \mathrm{MeV}<E_{\gamma}<1 \mathrm{GeV}$, the $\gamma p \rightarrow \pi N$ reaction involves many schannel contributions analogous to those of Figs. 16 and 17 for the $\Delta(1232)$. To indicate the state of the art in these descriptions, we quote the work of 
Ref. [29]. In this calculation, the $\gamma p \rightarrow \pi^{+} n$ reaction is studied for incident photon energies ranging from 400 till $1100 \mathrm{MeV}$. Besides the $\Delta(1232)$, five intermediate baryon resonances are taken into account: the $N^{*}(1440)$, the $N^{*}(1520)$, the $N^{*}(1535)$, the $N^{*}(1680)$ and the $\Delta^{*}(1700)$. The role of these contributions is shown in Fig. 20. The data are from Ref. [30]. It is clear that many resonances build up the pion photoproduction cross section in that energy range, making it hard to extract detailed information from the data.
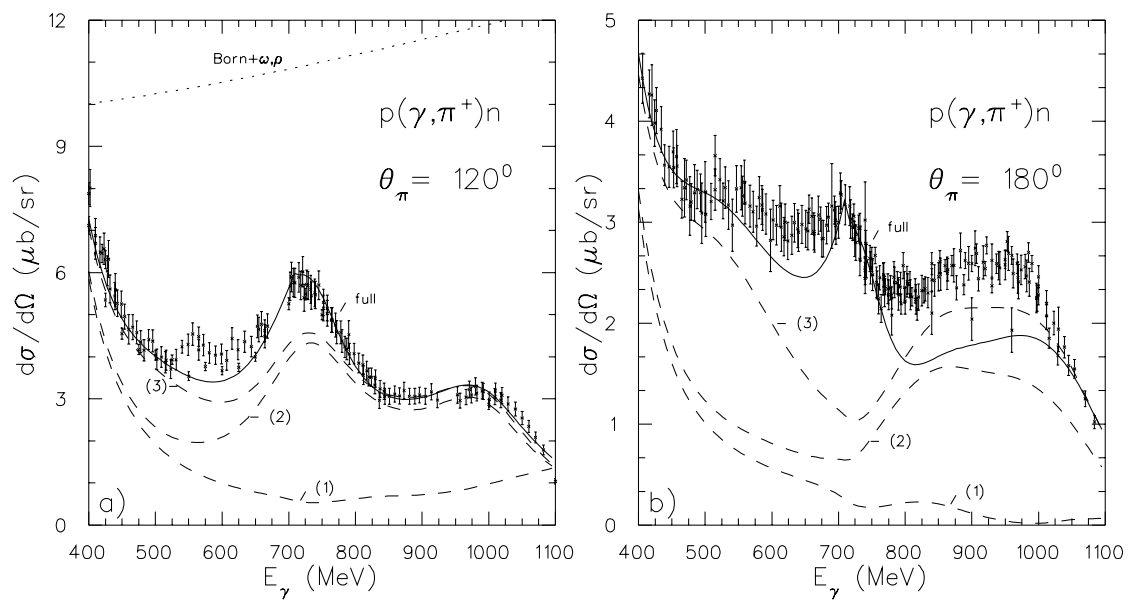

Figure 20. Differential cross sections for the $\gamma p \rightarrow \pi^{+} n$ reaction at a) $\theta_{\pi}=120^{\circ}$ and b) $\theta_{\pi}=180^{\circ}$ (from Ref. [29]). The dotted curve is the nucleon s-channel and vector meson t-channel contributions. The dashed curves are the results obtained with subsequent addition of baryon resonances:(1) with the $\Delta(1232)$, (2) (1) $+\mathrm{N}^{*}(1520)+\mathrm{N}^{*}(1680)+$ $\Delta^{*}(1700)$, (3) (2) $+N^{*}(1440)$. The solid curves are the full calculations which include the $\mathrm{N}^{*}(1535)$ resonance. The data are from Ref. [30].

\subsection{THE PHOTOPRODUCTION OF $\eta$-MESONS}

The $\gamma p \rightarrow \eta p$ reaction near threshold $\left[\mathrm{E}_{\gamma}^{\text {thresh }}=0.707 \mathrm{GeV}\right]$ is a very interesting process because it is largely dominated by only one baryon resonance, the $\mathrm{N}^{*}(1535)$. The amplitude for the $\eta$-meson photoproduction from protons will therefore be described by t-channel $\rho$ - and $\omega$-exchange diagrams and s-channel nucleon and $\mathrm{N}^{*}(1535)$ graphs. To have a quantitative feeling for the $\mathrm{N}^{*}(1535)$ dominance in the $\gamma p \rightarrow \eta p$ reaction, we quote the calculation of Ref. [31]. The $\gamma p \rightarrow \eta p$ reaction is computed in a unitary and gauge invariant model with coupled pion-nucleon and $\eta$-nucleon channels. Besides the terms mentioned above, the $\mathrm{N}^{*}(1650)$ is also included 
in the calculation. The result for the total cross section of the $\gamma p \rightarrow \eta p$ reaction for $\mathrm{E}_{\gamma}<1 \mathrm{GeV}$ is displayed in Fig 21. The data are from Ref. [32].

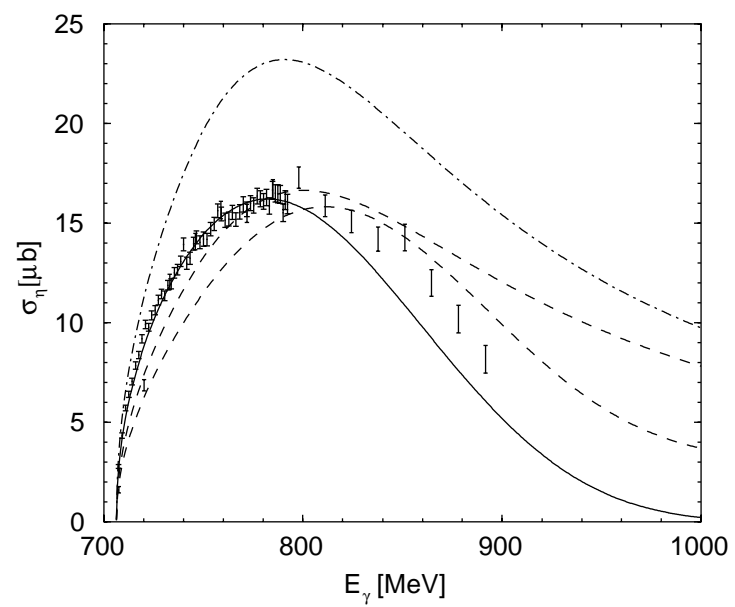

Figure 21. Total cross sections for the $\gamma p \rightarrow \eta p$ reaction near threshold (from Ref. [31]). The data are from Ref. [32]. The solid curve shows the result of the full calculation. The upper dashed line gives the cross section when all processes except those involving the $N^{*}(1535)$ resonance are neglected. The dot-dashed line is obtained by adding the non-resonant pion-nucleon Born terms and the lower dashed line by including the $\mathrm{N}^{*}(1650)$ resonance.

The contribution from the $\mathrm{N}^{*}(1535)$ resonance only seems to provide a rather good fit to the data. There are large cancellations among the other processes. The $\mathrm{N}^{*}(1650)$ resonance is not very strongly coupled to the $\eta \mathrm{N}$ channel but appears to affect the $\gamma p \rightarrow \eta p$ amplitude through the coupling between $\eta \mathrm{N}$ and $\pi \mathrm{N}$ channels.

There is at present a consensus on the dominant role of the $\mathrm{N}^{*}(1535)$ resonance in the photoproduction of $\eta$-mesons from protons close to threshold. The importance of higher baryon resonances is under investigation in various models [33, 34].

\subsection{THE PHOTOPRODUCTION OF $\eta$ '-MESONS}

Very little is known about the $\gamma p \rightarrow \eta^{\prime} p$ reaction near threshold $\left[\mathrm{E}_{\gamma}^{\text {thresh }}=\right.$ $1.447 \mathrm{GeV}]$. It has been measured recently by the SAPHIR Collaboration at Bonn [35]. The error bars are very large. The data seem consistent with two s-channel resonances with masses of the order of 1.9 and $2 \mathrm{GeV}$. New and better data on this process would open the way for very interesting theoretical investigations. 


\section{The associated photoproduction of kaons and hyperons $(\Lambda, \Sigma)$}

The associated photoproduction of kaons and hyperons $(\Lambda, \Sigma)$ near threshold $\left[\mathrm{E}_{\gamma}^{\text {thresh }}=0.911 \mathrm{GeV}\right.$ for the $\gamma p \rightarrow K^{+} \Lambda$ reaction, $\mathrm{E}_{\gamma}^{\text {thresh }}=1.048 \mathrm{GeV}$ for the $\gamma p \rightarrow K^{0} \Sigma^{+}$reaction] appears as a rather involved process. The Feynman graphs contributing to the amplitude of the $\gamma p \rightarrow K Y$ reaction near threshold are displayed in Figs. 22-25.

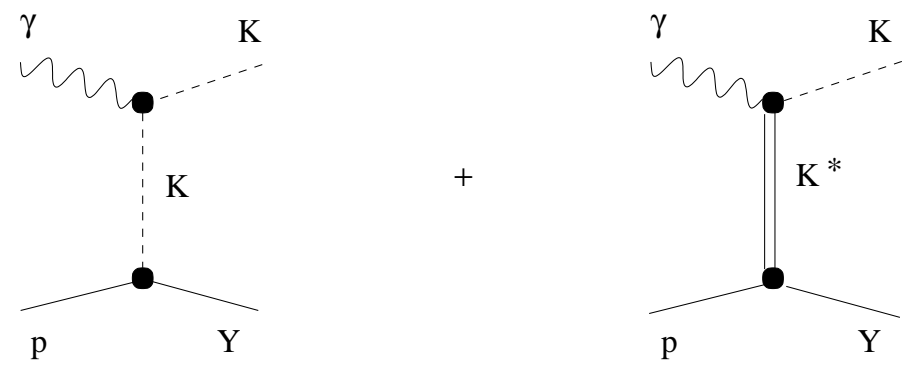

Figure 22. t-channel $\mathrm{K}$ and $\mathrm{K}^{*}$ pole terms for the $\gamma p \rightarrow K Y$ reaction near threshold.

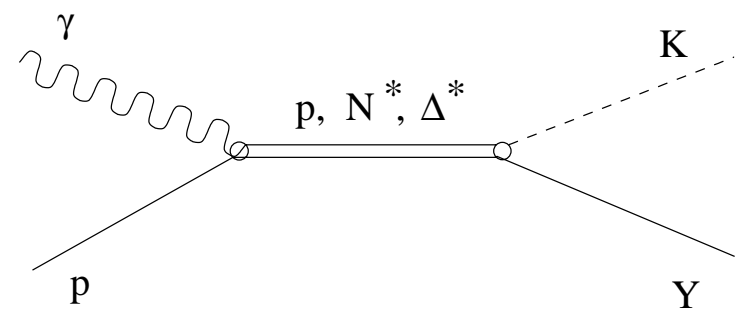

Figure 23. Direct s-channel exchange contributions to the $\gamma p \rightarrow K Y$ reaction near threshold.

The t-channel graphs of Fig. 22 are analogous to the pion pole and vector-meson exchange terms of $\pi^{+}$photoproduction. Their calculation involves more uncertainty however because, besides the kaon-exchange, both $\mathrm{K}^{*}(892)$ - and $\mathrm{K}^{*}(1270)$-exchanges play a role near threshold $\left(\mathrm{E}_{\gamma}<2.2 \mathrm{GeV}\right)$ $[36,37,38]$. The corrresponding vertices need to be parametrized.

In the s-channel (Figs. 23 and 24), in contrast to the situation discussed previously for the $\gamma p \rightarrow \eta p$ reaction, there is no prominent baryon resonance and this will be also a source of theoretical uncertainties. Many states are needed $[36,37,38]$ in the direct channel to explain the available data [39]. We show in Fig. 26 the calculation of Ref. [36] to illustrate this point. The resonances included are the $\mathrm{N}^{*}(1650)$, the $\mathrm{N}^{*}(1710)$ and the $\mathrm{N}^{*}(1720)$ 


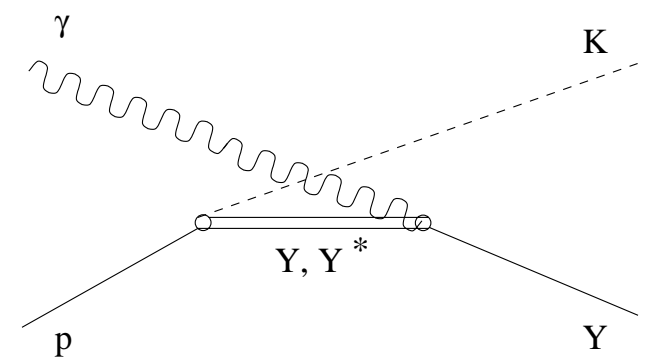

Figure 24. Crossed s-channel exchange contributions to the $\gamma p \rightarrow K Y$ reaction near threshold.

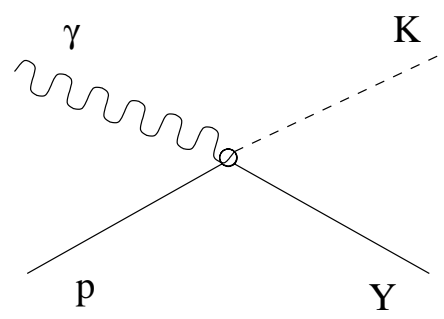

Figure 25. Contact term contribution to the $\gamma p \rightarrow K Y$ reaction near threshold.

for the $K \Lambda$ channel and, in addition, the $\Delta^{*}(1900)$ and the $\Delta^{*}(1910)$ for the $K \Sigma$ channel. The difference between the dash-dotted and solid curves shows the role of the s-channel graphs involving these resonances. It is most important. The excess of cross section around $1.9 \mathrm{GeV}$ for the $\gamma p \rightarrow$ $K^{+} \Lambda$ reaction has lead to the suggestion that there could be an additional resonance in this region [40].

In view of the complexity of the $\gamma p \rightarrow K Y$ reaction, many detailed observables (such as polarization data) will be needed in order to pin down specific processes.

\section{Concluding remarks}

A few directions emerge from this review of meson photoproduction from proton targets.

There is much interesting hadronic physics to be learnt from these reactions, particularly close to the production threshold. This kinematic region is the most likely to be described by a small number of diagrams and to allow the determination of hadron vertices. 


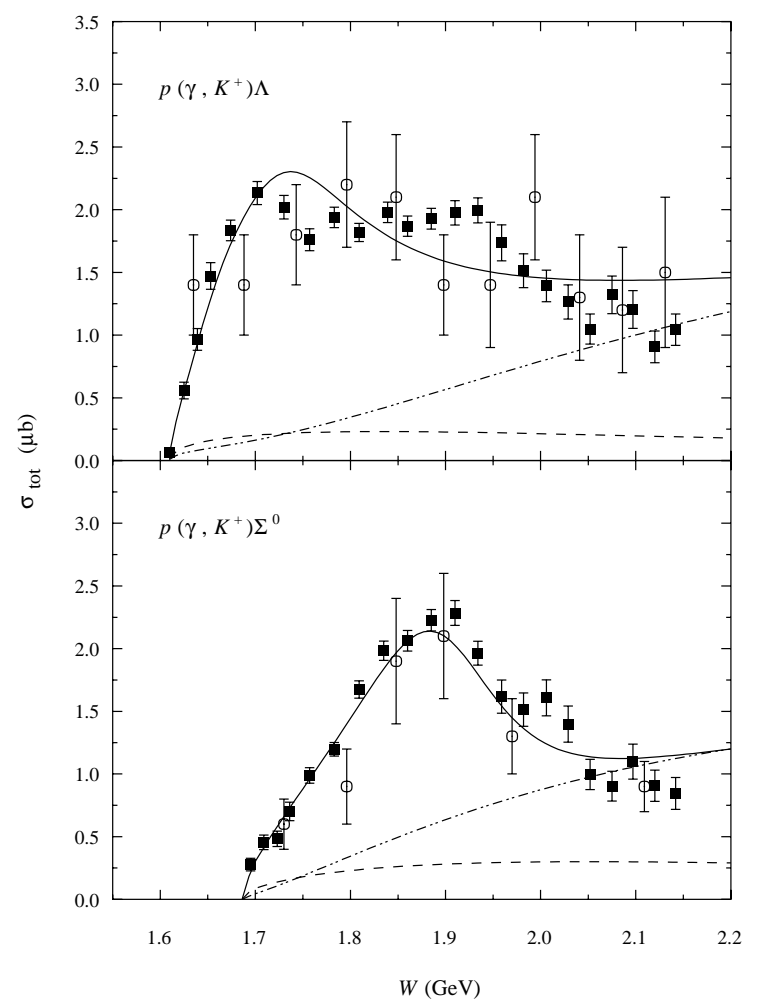

Figure 26. Total cross sections for the $\gamma p \rightarrow K^{+} \Lambda$ and $\gamma p \rightarrow K^{+} \Sigma$ reactions near threshold (from Ref. [36]). The data are from Ref. [39]. The solid curve shows the result of the full calculation. The dashed line gives the contribution of the t-channel K-exchange diagram, contact term and nucleon and hyperon s-channel graphs. The dash-dotted line indicates the effect of adding the t-channel $\mathrm{K}^{*}$-exchange diagrams.

Ongoing experimental programs should bring soon new and accurate data able to put more stringent constraints on theoretical models. This is especially the case for the photoproduction of $\rho$-, $\omega$ - and $\phi$-mesons. These three processes appear rather different and their detailed understanding is very much needed, particularly in view of the relation of vector meson fields to the electromagnetic current through the Vector Dominance Model. Another especially interesting process is the photoproduction of $\eta^{\prime}$-mesons, for which very few data are at present available.

Detailed studies of pion and kaon photoproduction are now becoming available. They are useful not only to study baryon resonances, but also aspects of Chiral Perturbation Theory very close to threshold. They are also the best processes to evaluate final state interactions. 


\section{Acknowledgements and apologies}

The author thanks the organizers for inviting her to lecture at Nijmegen and for waiting patiently for her manuscript. She apologizes to all those who made important contributions to the field of meson photoproduction near threshold and whose work is not quoted among the references. 


\section{References}

1. G. Veneziano, Phys. Rep. 9 C (1973) 199.

2. G. Veneziano, Nuovo Cimento 57 A (1968) 190.

3. N.M. Kroll, T.D. Lee and B. Zumino, Phys. Rev. 157 (1967) 1376.

4. Review of Particle Physics, Eur. Phys. J. C 3 (1998) 1.

5. J.J. Sakurai, Currents and Mesons, The University of Chicago Press, 1969.

6. L.G. Landsberg, Phys. Rep. 128 (1985) 30.

7. M. Soyeur, Acta Physica Polonica B 27 (1996) 401.

8. B. Friman and M. Soyeur, Nucl. Phys. A 600 (1996) 477.

9. F. Iachello, A.D. Jackson and A. Lande, Phys. Lett. B 43 (1973) 191.

10. G.E. Brown, M. Rho and W. Weise, Nucl. Phys. A 454 (1986) 669.

11. F. Klingl, N. Kaiser and W. Weise, Z. Phys. A 356 (1996) 193.

12. Aachen-Berlin-Bonn-Hamburg-Heidelberg-München Collaboration, Phys. Rev. 175 (1968) 1669.

13. J. Ballam et al., Phys. Rev. D 7 (1973) 3150.

14. H.J. Besh et al., Nucl. Phys. B 70 (1974) 257.

15. F.J. Klein et al., PiN Newslett. 14 (1998) 141.

16. W.J. Briscoe et al., Fizika B 8 (1999) 11.

17. A. Titov, T.-S.H. Lee and H. Toki, Phys. Rev. C 59 (1999) 2993.

18. R. Machleidt, Adv. Nucl. Phys. 19 (1989) 189.

19. L. Tiator, C. Bennhold and S.S. Kamalov, Nucl. Phys. A 580 (1994) 455.

20. A.W. Thomas and K. Holinde, Phys. Rev. Lett. 63 (1989) 2025.

21. T. Ericson and W. Weise, Pions and Nuclei, Clarendon Press, Oxford, 1988.

22. O. Hanstein, D. Drechsel and L. Tiator, Nucl. Phys. A 632 (1998) 561.

23. F. Haerter, Ph.D. Thesis, Mainz (1996).

24. A2 Collaboration at MAMI, private communication.

25. T. Fuji et al., Phys. Rev. Lett. 28 (1972) 1672.

26. G. Chiefari et al., Lett. Nuovo Cim 13 (1975) 129.

27. R. M. Davidson et al., Phys. Rev. C 59 (1999) 1059.

28. U.-G. Meissner, Baryons'98, Proceedings of the 8th International Conference on the Structure of Baryons, 22-26 September 1998, Bonn, Germany, World Scientific Ed., p 135.

29. D. Drechsel et al., Nucl. Phys. A 645 (1999) 145.

30. R.A. Arndt, I.I. Strakovsky and R.L. Workman, Phys. Rev. C 53 (1996) 430.

31. C. Deutsch-Sauermann, B. Friman and W. Noerenberg, Phys. Lett. B 409 (1997) 51.

32. B. Krusche et al., Phys. Rev. Lett. 74 (1995) 3736.

33. N.C. Mukhopadhyay and N. Mathur, Phys. Lett. B 444 (1998) 7.

34. Z. Li and B. Saghai, Nucl. Phys. A 644 (1998) 345.

35. R. Ploetzke et al., Phys. Lett. B 444 (1998) 555.

36. F.X. Lee et al., nucl-th/9907119.

37. C. Bennhold et al., to be published in the Proceedings of the Workshop on ElectronNucleus Scattering, Elba, Italy, June 22-26, 1998.

38. J.C. David et al., Phys. Rev. C 53 (1996) 2613.

39. M.Q. Tran et al., Phys. Lett. B 445 (1998) 20.

40. T. Mart and C. Bennhold, nucl-th/9906096. 